\title{
ON THE HAUSDORFF OPEN CONTINUOUS IMAGES OF HAUSDORFF PARACOMPACT p-SPACES
}

\author{
H. H. WICKE ${ }^{1}$
}

1. Introduction. Ponomarev proved the following remarkable theorem: Every $T_{0}$ first-countable space of infinite cardinality is an open continuous image of a zero-dimensional metrizable space of the same weight $[8] .{ }^{2}$ This theorem clearly and succinctly summarizes the behavior of metrizable spaces under open mappings. The purpose of this article is to prove an analogue of Ponomarev's theorem in a not necessarily first-countable situation and to develop some of its consequences. This analogue, Theorem 1 below, is a joint discovery of the author and Dr. J. M. Worrell, Jr. [10]. Remark 4 shows how a proof of Ponomarev's theorem may be derived from the proof of Theorem 1. Theorem 1 leads directly to a characterization (Theorem 2) of the class of Hausdorff open continuous images of Hausdorff paracompact $p$-spaces as the class of Hausdorff spaces of pointcountable type. The latter class generalizes the class of Hausdorff first-countable spaces. Both the concept of $p$-space and of space of point-countable type are due to Arhangel'skir [3], [4]. Theorem 3, a rather direct consequence of Theorem 1, answers a question of Arhangel'skil by generalizing a theorem of his to the Hausdorff case. A relation between Theorem 1, which involves single-valued mappings, and Theorem 3, which involves many-valued mappings, is pointed out in Remark 3.

2. Terminology. The general terminology used here is much like that of [7], one exception being that spaces called compact in [7] are here called bicompact. The usage of [7] in letting $X$ ambiguously denote the topological space $(X, J)$ is followed where convenient, and product space refers to a Cartesian product of spaces endowed with the product topology [7]. A base for $X$ means a base for the topology of $X$. The letter $N$ denotes the set of positive integers and if $A$ is a set, $\aleph(A)$ denotes the cardinal number of $A$. The weight [2] of a topological space $(X, \Im)$ is defined as the smallest cardinal num-

Presented to the Society, April 19, 1969 under the title On a problem of Arhangel'skix; received by the editors June 24, 1968.

1 This work was supported by the United States Atomic Energy Commission.

2 Ponomarev does not point out that infinite cardinality is required. In fact, if $S$ is a finite $T_{0}$ but not $T_{1}$ space any $T_{1}$ open continuous preimage of $S$ has infinite weight and cardinality. In Theorem 1 infinite cardinality is not required since the spaces here are assumed to be $T_{\mathbf{2}}$. 
ber $m$ such that $\mathfrak{J}$ has a base of cardinal $m$. A mapping $f: X \rightarrow Y$ is called perfect [1] if and only if it is closed, continuous, and $f^{-1}(y)$ is bicompact for all $y \in Y$. If $a$ is a collection of sets then $\operatorname{St}(x, a)$ denotes $U\{A \in a: x \in A\}$. A $T_{1}$-space $X$ is called a $p$-space [3] if and only if there exists a sequence $\mathcal{G}_{1}, \mathcal{G}_{2}, \cdots$ of collections of open subsets of the Wallman bicompactification $\omega X$ of $X$ covering $X$ such that if $x \in X, \cap\left\{\operatorname{St}\left(x, g_{n}\right): n \in N\right\} \subset X$. If $X$ is a Tychonoff space this definition is equivalent to one in which $\beta X$ (the Stone-Cech bicompactification of $X$ ) replaces $\omega X$. A principal theorem for $p$-spaces, suggestive of the naturality of their use in Theorem 1 , is that of Arhangel'skili: $A T_{2}$-space is a paracompact p-space if and only if there exists a perfect mapping of it onto a metrizable space [3, Theorem 5.1].

3. Theorems. If $(X, J)$ is a space and $A \subset X$, a subcollection $D$ of $J$ whose members include $A$ is called a base at $A$ if and only if for every $U \in J$ such that $U \supset A$, there exists $D \in D$ such that $A \subset D \subset U$.

If $X$ is a space and $A \subset X$, then $A$ is said to be of countable character [4] if and only if there exists a countable base at $A$.

A space $X$ is said to be of point-countable type [4] if and only if $X$ is covered by a collection of bicompact subspaces of countable character.

REMARK 1. Any first-countable space is of point-countable type.

REMARK 2. The property of being of point-countable type is preserved by open continuous mappings.

The following lemma was stated by Arhangel'skir [5, p. 158]. A proof is sketched here for completeness.

Lemma 1. A Tychonoff p-space is of point-countable type.

Proof. Every point of such a space $X$ lies in a bicompact subset of $X$ which is a $G_{\delta}$-set in $\beta X$ and every such set has countable character.

Lemma 2. In a Hausdorff space $X$ the following properties are equivalent:

(i) $X$ is of point-countable type.

(ii) If $U$ is open in $X$ and $x \in U$ there exists a bicompact set $B$ of countable character such that $x \in B \subset U$.

Proof. Clearly (ii) implies (i). Suppose $x \in U$ and $U$ is open. There exists a bicompact set $B$ of countable character containing $x$. Let $\left\{U_{k}: k \in N\right\}$ be a base at $B$ such that $U_{k+1} \subset U_{k}$ for all $k \in N$. Then since $X$ is Hausdorff $B=\bigcap\left\{\bar{U}_{k}: k \in N\right\}$. Let $V_{1}=U$. Suppose open sets $V_{1}, \cdots, V_{n}$ have been defined such that $x \in V_{k} \subset U_{k} \cap V_{k-1}$ and $\bar{V}_{k}$ is disjoint from $B \sim V_{k-1}$ for $1<k \leqq n$. Since $B \sim V_{n}$ is bicompact, $x \in V_{n}$ and $X$ is $T_{2}$, there exists an open set $V$ such that $x \in V \subset \bar{V} \subset X$ $\sim\left(B \sim V_{n}\right)$. Let $V_{n+1}=V \cap V_{n} \cap U_{n+1}$. Thus there exists a sequence 
$\left\{V_{n}\right\}$ such that for all $n \in N, x \in V_{n+1} \subset V_{n} \cap U_{n+1}$ and $\bar{V}_{n+1}$ is disjoint from $B \sim V_{n}$. Let $C=\cap\left\{\bar{V}_{n}: n \in N\right\}$. Then $C$ is a closed (therefore bicompact) subset of $B$ containing $x$. Since $\bar{V}_{n+1} \subset(X \sim B) \cup V_{n}, C$ $=\bigcap\left\{V_{n}: n \in N\right\}$ and $C \subset U$. Suppose $W$ is open and $C \subset W$. If no $V_{n} \subset W$, there exists a sequence $\left\{x_{k}\right\}$ such that each $x_{k} \in V_{k} \sim W$. Since $\bigcap\left\{\bar{V}_{k} \sim W: k \in N\right\}=\varnothing$ and $B$ is bicompact, there exists $n$ such that $\bar{V}_{k} \sim W \subset X \sim B$ for all $k \geqq n$. Let $A=\left\{x_{k}: k \geqq n\right\}$. Then $\bar{A} \subset X$ $\sim W$ and $\bar{A} \cap B \neq \varnothing$. For if $B \subset X \sim \bar{A}$, then for some $k \geqq n, U_{k} \subset X$ $\sim \bar{A} \subset X \sim A$ contradicting $x_{k} \in A$. If $y \in \bar{A} \cap B, y \in \bar{V}_{k} \sim W$ for all $k \in N$, again a contradiction. Hence some $V_{n} \subset W$, so that $C$ has countable character.

Theorem 1. Suppose $X$ is a Hausdorff space of point-countable type. Then $X$ is the range of an open continuous mapping $\phi$ such that: (1) The domain $Y$ of $\phi$ is a Hausdorff paracompact p-space. (2) The weight of $Y$ is the weight of $X$. (3) $Y$ is a subspace of the product space of a zerodimensional metrizable space and $X$.

Proof. See $\$ 4$.

Comment. For Tychonoff spaces, part (1) can be derived from [4, Theorem 3.14] by the method of Remark 3 below.

TheOREM 2. A Hausdorff space is of point-countable type if and only if it is an open continuous image of a Hausdorff paracompact p-space.

Proof. This follows from Theorem 1, Lemma 1, and Remark 2.

Recall that a many-valued mapping $f: X \rightarrow Y$ is called continuous (from above) [9] if and only if for every $x \in X$ if $V \subset Y$ is open and $f x \subset V$ there exists an open $U \subset X$ such that $x \in U$ and $f(U) \subset V$. The mapping $f$ is called range-bicompact (or $Y$-bicompact [9]) if and only if $f x$ is bicompact for every $x \in X$. Arhangel'skil proved the following theorem with the additional hypothesis that $X$ is a Tychonoff space $[4$, Theorem 3.14] and asked $[4$, p. 54$]$ whether it is valid for a wider class of spaces.

Theorem 3. Suppose $X$ is a Hausdorff space. Then $X$ is of pointcountable type if and only if $X$ is the range of an open continuous (possibly many-valued) range-bicompact mapping of a metrizable space.

Proof. By Theorem 1, there exists a continuous mapping $\phi$ of a $T_{2}$ paracompact $p$-space $Y$ onto $X$. By Arhangel'skii's theorem (see $\$ 2$ ) there exists a perfect mapping $\theta$ of $Y$ onto a metrizable space $M$. It is straightforward to show that $\phi \circ \theta^{-1}$ is an open continuous rangebicompact mapping of $M$ onto $X$. The sufficiency follows from [4, Proposition 3.6]. 
REMARK 3. Theorem 3 can be used to derive part (1) of Theorem 1. For if $f$ is an open continuous many-valued range-bicompact mapping of a metrizable space $X$ onto a Hausdorff space $Y$ of point-countable type, let $Z=\{(x, y) \in X \times Y: y \in f x\}$, under the topology induced by the product topology. The set $Z$ is called the graph of $f$ by Ponomarev [9]. If $\theta$ and $\phi$ denote the projections of $Z$ onto $X$ and $Y$ respectively, then it may be seen that $f=\phi \circ \theta^{-1}$ where $\phi$ is open and continuous and $\theta$ is perfect. (This statement may be proved in a fashion similar to that used by Ponomarev in showing that a perfect mapping $f$ factors in to $\phi \circ \theta^{-1}$ where $\theta$ and $\phi$ are perfect [9, Theorem $1, \S 2]$.) Hence $Z$ is a paracompact $p$-space by Arhangel'skil's theorem and $\phi$ maps $Z$ onto $Y$.

\section{Proof of Theorem 1 .}

Proof. Assume $\boldsymbol{N}(X)$ is infinite. Let $\mathcal{C}$ denote $\{B C X: B$ is bicompact and of countable character $\}$. For some base $W$ of $X$ such that weight of $X=\boldsymbol{N}$ (W), let $\mathcal{F}$ denote the collection of all unions of finite subcollections of $W$. Then $\aleph(F)=$ weight of $X$ and $\mathscr{F} \subset \mathcal{F}$. Call a sequence $\alpha$ admissible if and only if for each $n \in N:(1) \alpha(n) \in \mathcal{F}$; (2) $\alpha(n+1) \subset \alpha(n)$; (3) for some $B \in \mathcal{C}, B=\bigcap\{\alpha(k): k \in N\}$ and $\{\alpha(k): k \in N\}$ is a base at $B$. Using bicompactness it may be seen that for each $B \in \mathcal{C}$ there exists an admissible sequence $\alpha$ satisfying (3) with respect to $B$.

Consider $\mathcal{F}$ as a topological space with the discrete topology and let $\Delta$ denote the product space of countably many copies of F. Let $\Gamma=\{\alpha \in \Delta: \alpha$ is admissible $\}$. Then $\Gamma$ is a metrizable zerodimensional space (it is a subspace of a Baire space [6]). Let $\Gamma \times X$ denote the product space of $\Gamma$ and $X$ and let

$$
Y=\{(\alpha, x) \in \Gamma \times X: x \in \cap\{\alpha(k): k \in N\}\},
$$

with the topology induced by the product topology. Note that $Y$ is Hausdorff. Let $\theta=\pi_{1} \mid Y$ and $\phi=\pi_{2} \mid Y$, where $\pi_{i}$ denotes projection on to the $i$ th coordinate. Then $\theta$ and $\phi$ are continuous mappings of $Y$ onto $\Gamma$ and $X$ respectively.

If $\alpha \in \Gamma$, let $S(\alpha \mid n)=\left\{\alpha^{\prime} \in \Gamma: \alpha^{\prime}(k)=\alpha(k), k=1, \cdots, n\right\}$. Then $\{S(\alpha \mid n): n \in N$ and $\alpha \in \Gamma\}$ is a base for $\Gamma$. For $\alpha \in \Gamma$ and $V \in \mathcal{F}$ such that $V \subset \alpha(n)$ let $D(\alpha \mid n ; V)$ denote $(S(\alpha \mid n) \times V) \cap Y$. Then $B$ $=\{D(\alpha \mid n ; V): \alpha \in \Gamma, n \in N, V \in \mathcal{F}$, and $V \subset \alpha(n)\}$ is a base for $Y$. Since $\boldsymbol{\aleph}(\mathcal{F})=$ weight of $X, \boldsymbol{N}(B)=$ weight of $X$.

Suppose $\alpha \in \Gamma, V \in F$, and $V \subset \alpha(n)$. Then clearly $\phi[D(\alpha \mid n ; V)]$ $\subset V$. If $x \in V$, then by Lemma 2 there exists $B \in \mathcal{C}$ such that $x \in B$ $\subset V$. Let $\beta \in \Gamma$ be such that $\{\beta(k): k \in N\}$ is a base at $B$. There exists $k$ such that $\beta(k) \subset V$. The sequence $\alpha^{\prime}$ such that $\alpha^{\prime}(j)=\alpha(j), 1 \leqq j \leqq n$ 
and $\alpha^{\prime}(j)=\beta(k+j)$ for $j>n$ is admissible and $\left(\alpha^{\prime}, x\right) \in D(\alpha \mid n ; V)$. Hence $\phi[D(\alpha \mid n ; V)]=V$. Therefore $\phi$ is an open mapping.

If it is shown that $\theta$ is a perfect mapping, then by Arhangel'skir's theorem cited in $\S 2, Y$ is a paracompact $p$-space. Suppose $\alpha \in \Gamma$ and $B=\bigcap\{\alpha(k): k \in N\}$. Then, since $B$ is bicompact, $\theta^{-1}(\alpha)=\{\alpha\} \times B$ is bicompact. Hence $\theta$ is a bicompact mapping. To show that $\theta$ is closed suppose $W$ is open in $Y$ and $\theta^{-1}(\alpha) \subset W$. There exist $m \in N$ and sets $D_{k}=D\left(\alpha_{k} \mid n(k) ; V_{k}\right) \in 囚$ intersecting $\theta^{-1}(\alpha)$ for $k=1, \cdots, m$, such that $\theta^{-1}(\alpha) \subset \cup\left\{D_{k}: k \leqq m\right\} \subset W$. Since $\theta^{-1}(\alpha)$ meets each $D_{k}, \alpha_{k}(j)$ $=\alpha(j), 1 \leqq j \leqq n(k), 1 \leqq k \leqq m$. Also $B \subset \cup\left\{V_{k}: k \leqq m\right\}$. By conditions (2) and (3) on admissible sequences there exists $n \geqq \max \{n(k): k \leqq m\}$ such that $B \subset \alpha(n) \subset \bigcup\left\{V_{k} ; k \leqq m\right\}$. If $\left(\alpha^{\prime}, x\right) \in D=D(\alpha \mid n ; \alpha(n))$, then $x \in V_{k}$ for some $k$, and therefore $\left(\alpha^{\prime}, x\right) \in D_{k} \subset W$. Hence $\theta^{-1}(\alpha)$ $\subset D \subset W$. Since any $\theta^{-1}\left(\alpha^{\prime}\right)$ intersecting $D$ is a subset of $D, D=\theta^{-1} \theta(D)$. It follows that $\theta$ is a closed mapping.

REMARK 4. If the space $X$ is $T_{0}$ and first-countable, then $\mathcal{C}$ in the above proof can be taken as the collection $\{\{x\}: x \in X\}$. Then each admissible sequence $\alpha$ is such that $\bigcap\{\alpha(k): k \in N\}=\{x\}$ for some $x \in X$. It follows that $Y$ is homeomorphic to $\Gamma$ and thus $X$ is an open continuous image of $\Gamma$. This proves Ponomarev's theorem.

\section{REFERENCES}

1. P. S. Aleksandrov (=P. S. Alexandroff), On certain results in the theory of topological spaces obtained in the last twenty-five years, Uspehi Mat. Nauk 15 (1960), No. 2(92), 25-95 = Russian Math. Surveys 15 (1960), No. 2, 23-83.

2. P.S. Aleksandrov and P. S. Uryson (=P. S. Urysohn), Mémoire sur les espaces topologiques compacts, Verh. Nederl. Akad. Wetensch. Afd. Natuurk. Sect. I 14, No. 1 (1929), 1-96.

3. A. V. Arhangel'skir, On a class of spaces containing all metric and all locally bicompact spaces, Mat. Sb. 67 (1965), 55-85. (Russian)

4. - Bicompact sets and the topology of spaces, Trudy Moskov. Mat. Obšx. 13 (1965), 3-55 = Trans. Moscow Math. Soc. 1965, 1-62.

5. - Mappings and spaces, Uspehi Mat. Nauk. 21 (1966), No. 4(130), 133$184=$ Russian Math. Surveys 21 (1966), No. 4, 115-162.

6. F. Hausdorff, Mengenlehre, 3rd ed., Gruyter, Berlin-Leipzig, 1935.

7. J. L. Kelley, General topology, Van Nostrand, Princeton, N. J., 1955.

8. V. I. Ponomarev, Axioms of countability and continuous mappings, Bull. Acad. Polon. Sci., Sér. Sci. Math. Astronom. Phys. 8 (1960), 127-133. (Russian)

9. - Properties of topological spaces preserved under multivalued continuous mappings, Mat. Sb. 51(93) (1960), 515-537; English transl., Amer. Math. Soc. Transl. (2)38 (1964), 119-140.

10. J. M. Worrell, Jr. and H. H. Wicke, Concerning a class of completely regular spaces described by Arhangel'skiน, Notices Amer. Math. Soc. 15 (1968), 352.

Sandia laboratories, Albuquerque, New Mexico 